EPJ Web of Conferences 66, 04009 (2014)

DOI: 10.1051/epjconf/20146604009

(C) Owned by the authors, published by EDP Sciences, 2014

\title{
Anisotropic Flow from Non-equilibrium Initial Condition with a Saturation Scale
}

\author{
V. Greco ${ }^{1,2, a}$, M. Ruggieri ${ }^{1}$, F. Scardina ${ }^{1}$, S. Plumari ${ }^{1,2}$, and A. Puglisi ${ }^{1,2}$ \\ ${ }^{1}$ Department of Physics and Astronomy, University of Catania, Via S. Sofia 64, I-95125 Catania (Italy) \\ ${ }^{2}$ Laboratorio Nazionale del Sud, INFN-LNS, Via S. Sofia 63, I-95125 Catania (Italy)
}

\begin{abstract}
A current goal of relativistic heavy ion collisions experiments is to understand the impact of initial non-equilibrium on final observables. A Color Glass Condensate (CGC) as the limiting state of QCD matter at very high density implies initial non-thermal distribution at least for momenta below the saturation scale. In viscous hydrodynamics simulations, a standard Glauber initial condition leads to estimate $4 \pi \eta / s \sim 1$, while employing the Kharzeev-Levin-Nardi (KLN) modeling of the CGC leads to at least a factor of 2 larger $\eta / s$. Within a kinetic theory approach based on a relativistic Boltzmann-like transport simulation, our main result is that the out-of-equilibrium initial distribution in p-space reduces the efficiency in building-up the elliptic flow. At RHIC energy we find the available data on $v_{2}$ are in agreement with a $4 \pi \eta / s \sim 1$ also for KLN initial conditions.
\end{abstract}

\section{Introduction}

A main discovery of the Relativistic Heavy-Ion Collider (RHIC) confirmed by the Large Hadron Collider (LHC) has been that the QGP has a very small shear viscosity to density entropy [1], $\eta / s$, close to the lower bound of $1 / 4 \pi$ conjectured for systems at infinite strong coupling. A key observable to reach such a conclusion is the so-called elliptic flow, $v_{2}=\left\langle\cos \left(2 \varphi_{p}\right)\right\rangle=\left\langle\left(p_{x}^{2}-p_{y}^{2}\right) /\left(p_{x}^{2}+p_{y}^{2}\right)\right\rangle$, with $\varphi_{p}$ being the azimuthal angle in the transverse plane and the average meant over the particle distribution. In fact, the expansion of the created matter generates a large anisotropy of the emitted particles that can be primarily measured by $v_{2}$. Its main origin in non-central collisions is the initial spatial eccentricity, $\epsilon_{x}=\left\langle x^{2}-y^{2}\right\rangle /\left\langle x^{2}+y^{2}\right\rangle$, of the overlap region. The observed large $v_{2}$ is considered a signal of a very small $\eta / s$ because it means that the system is very efficient in converting $\epsilon_{x}$ into an anisotropy in the momentum space $v_{2}$, a mechanism that would be strongly damped in a system highly viscous that dissipates and smooths anisotropies [2-4]. Quantitatively both viscous hydrodynamics $[2,3,5,6]$, and transport Boltzmann-like approaches [7-10] agree in indicating an average $\eta / s$ of the QGP lying in the range $4 \pi \eta / s \sim 1-3$.

The uRHIC program offers also the tantalizing opportunity to explore the existence of an exotic state, namely the Color Glass Condensate (CGC). Such a state of matter would be primarily generated by the very high density of the gluon parton distribution function at low $x$ (parton momentum fraction), which triggers a saturation of the gluon distribution function at a $p_{T}$ below the saturation scale, $Q_{s}$. In fact, a simple geometrical description through the Glauber model predicts a $\epsilon_{x}$ smaller at least $25-30 \%$

a e-mail: greco@lns.infn.it

This is an Open Access article distributed under the terms of the Creative Commons Attribution License 2.0, which permits unrestricted use, distribution, and reproduction in any medium, provided the original work is properly cited. 
than the eccentricity of the CGC $[11,12]$. According to viscous hydrodynamics, the experimental data of $v_{2}\left(p_{T}\right)$ at the highest RHIC energy are in agreement with a fluid at $4 \pi \eta / s \sim 1$, assuming a standard Glauber initial condition, while due to the larger $\epsilon_{x}$ MC-KLN favors instead a fluid at $4 \pi \eta / s \sim 2$.

\section{Transport approach at fixed viscosity}

The implementation of the melted CGC in hydrodynamics takes into account only the different $\mathrm{x}$ space distribution respect to a geometric Glauber model, discarding the key and more peculiar feature of the damping of the distribution for $p_{T}$ below the $Q_{s}$ saturation scale. We will show that instead this has a pivotal role on the build-up of $v_{2}$. To show the impact of a saturation scale in the initial distribution, we adopt the the factorized-KLN (fKLN) approach as introduced in $[11,12]$. We take the saturation scale for the nucleus $A$ as $Q_{s, A}^{2}\left(x, x_{\perp}\right)=1.3 \mathrm{GeV}^{2}\left(\frac{T_{A}\left(\boldsymbol{x}_{\perp}\right)}{p_{A}\left(\boldsymbol{x}_{\perp}\right)}\right)\left(\frac{0.01}{x}\right)^{\lambda}$, with $p_{A}\left(\boldsymbol{x}_{\perp}\right)=1-$ $\left(1-\sigma_{\text {in }} T_{A}\left(\boldsymbol{x}_{\perp}\right) / A\right)^{A}$, where $\sigma_{\text {in }}$ is the inelastic cross section and $T_{A}$ corresponds to the usual thickness function of the Glauber model. $\lambda=0.28$, and similarly nucleus $B$. This leads to a $\left\langle Q_{s}\right\rangle \approx 1.4 \mathrm{GeV}$ for central collisions, where the average is understood in the transverse plane.

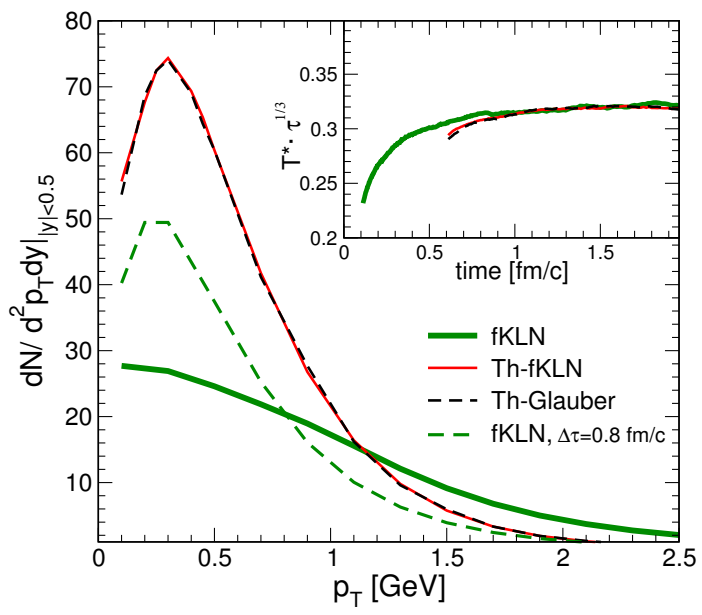

Figure 1. Momentum space distributions in a cylinder of radius $r=3 \mathrm{fm}$ and space rapidity $\left|\eta_{s}\right| \leq 1$ for the case of fKLN model at the initial time (thick solid line) compared to the the thermal one (thin solid and dashed lines) at their initial evolution time $\tau_{0}$ and to the one of fKLN after a dynamical evolution $\Delta \tau=0.8 \mathrm{fm} / \mathrm{c}$. Inset: time evolution of $\mathrm{T} \cdot \tau^{1 / 3}$ for the three different initial conditions considered. All the panels refer to Au-Au collisions at $\sqrt{s}=200 \mathrm{GeV}$, with an impact parameter $b=7.5 \mathrm{fm}$.

We employ the relativistic Boltzmann transport equation as a base of a simulation code of the fireball expansion created in relativistic heavy-ion collision [7, 10]. At variance with the standard use of transport theory, we have developed an approach that, instead of focusing on specific microscopic calculations or modelings for the scattering matrix, fixes the total cross section in order to have the wanted $\eta / s$. This can be achieved by mean of the Chapmann-Enskog approximation which has been shown to be in agreement with the results obtained using the Green Kubo formula [13]. Therefore, we fix $\eta / s$ and compute the local total cross section by mean of the relation $\sigma_{t o t}=\frac{1}{15} \frac{\langle p\rangle}{\rho g(a)} \frac{1}{\eta / s}$, which is valid for a generic differential cross section $d \sigma / d t \sim \alpha_{s}^{2} /\left(t-m_{D}^{2}\right)^{2}$. In the above equation $a=T / m_{D}$, with $m_{D}$ the screening mass regulating the angular dependence of the cross section, while

$$
g(a)=\frac{1}{50} \int d y y^{6}\left[\left(y^{2}+\frac{1}{3}\right) K_{3}(2 y)-y K_{2}(2 y)\right] h\left(\frac{a^{2}}{y^{2}}\right),
$$

with $K_{n}$ the Bessel function and $h$ corresponding to the ratio of the transport and the total cross section. A smaller value of $g(a)$ means that a higher $\sigma_{\text {tot }}$ is needed to reproduce the same value of $\eta / s$, because 
the cross section is more forward-backward peaked. We have checked that in the regime were viscous hydrodynamic applies (not too large $\eta / s$ and $p_{T}$ ) the specific microscopic detail of the cross section is irrelevant.

In the following, we will consider three different types of initial distribution function in the phasespace, two of which are the one employed in hydrodynamics, while the third one is the novelty of the present study. For $A u+A u$ collision at $\sqrt{s}=200 A G e V$ and $b=7.5 \mathrm{fm}$ our result for initial eccentricity in the fKLN model is $\epsilon_{x}=0.357$ (which is in agreement with MC-KLN [11] result used in hydro simulations). The standard initial condition for simulations of the plasma fireball created at RHIC is a $\boldsymbol{x}$-space distribution given by the Glauber model and a $\boldsymbol{p}$-space thermalized spectrum in the transverse plane at a time $\tau=0.6 \mathrm{fm} / \mathrm{c}$. In this case, for a standard mixture of $N_{\text {part }}$ and $N_{\text {coll }}$ we find $\epsilon_{x}=0.284$. We will refer to this case as $T h-f K L N$. Instead we will refer to $T h-G l a u b e r$ when in $\boldsymbol{x}$-space the distribution is given by the fKLN (or MC-KLN), while in the momentum space the spectrum is thermal at $\tau_{0} \sim 0.6 \mathrm{fm} / \mathrm{c}$; this is how $\mathrm{KLN}$ is implemented in hydrodynamics, leading to the conclusion that the CGC suggests a $4 \pi \eta / s \sim 2[5,11,14]$. The third initial conditions is the full fKLN initial conditions where, beyond the $\boldsymbol{x}$-space, the saturated distribution in $\boldsymbol{p}$-space is implemented as well, see Fig. 1 solid thick line. As initial time we take $\tau_{0}=0.15 \mathrm{fm} / \mathrm{c}$ because in this case there is no pre-assumption of thermalization. This is not usually considered in hydrodynamics because there it is implicitly assumed a distribution function in $p$-space in local equilibrium, at least in the transverse plane.

\section{Results}

In Fig. 1 we plot the initial spectra for the fKLN (thick solid line), Th-fKLN (dashed line) and ThGlauber (thin solid line) at their respective initial times $\tau_{0}$, and the spectrum of the fKLN model after a time evolution $\Delta \tau=0.8 \mathrm{fm} / \mathrm{c}$ (dashed green line). We notice that initially the fKLN spectrum is quite far from a thermalized spectrum; in fact, it embeds the saturation effects. Nevertheless, the spectrum evolves to a thermalized one within $1 \mathrm{fm} / \mathrm{c}$. Such a feature is confirmed by the inset of Fig. 1 , where the quantity $T^{*} \cdot \tau^{1 / 3}$ is shown, with $T^{*}=E / 3 N$ representing, the temperature in the case of a thermalized system. It is known that in the case of $1 \mathrm{D}$ expansion a thermalized system should keep such a ratio constant. We find that in the case of the fKLN (solid green line) the product $T^{*} \cdot \tau^{1 / 3}$ is initially strongly dependent on time because the system is quite far from equilibrium; however at $\tau \sim 0.8 \mathrm{fm} / \mathrm{c}$ both the value and the time evolution become indistinguishable from the thermal cases represented by the Th-Glauber and Th-fKLN. We have checked that also the ratio of longitudinal to transverse pressure $P_{L} / P_{T}$ gets close to unity at similar time.

In the left panel of Fig. 2 we plot the $v_{2}\left(p_{T}\right)$ for the case of Th-Glauber (thin solid line) and Th-fKLN (thick solid line) at a fixed $4 \pi \eta / s=1$. The Glauber initial condition reproduces quite well the data (circles); in the case of Th-fKLN (thick solid line) one gets a too large $v_{2}$ and for such initial conditions the agreement with the data is achieved only if the $\eta / s$ is increased by a factor of two (dashed line). These results are in agreement with the ones obtained from viscous hydrodynamics $[5,14]$, showing the solidity and consistency of our transport approach at fixed $\eta / s$. In the right panel of Fig. 2 we present our novel result for the fKLN model, when the CGC distribution function is implemented in both the $\boldsymbol{x}$ and $\boldsymbol{p}$ spaces. We find that fKLN with a $4 \pi \eta / s=1$ (thick solid line) gives a $v_{2}\left(p_{T}\right)$ quite similar to the Th-Glauber, while in such a case if $4 \pi \eta / s=2$, dashed line, the $v_{2}\left(p_{T}\right)$ would be too small. Our interpretation is that the initial larger $\epsilon_{x}$ is compensated by the key feature of an almost saturated initial distribution in $p$-space below the saturation scale $Q_{s}$. In other words the initial out-of-equilibrium fKLN distribution reduces the efficiency in converting $\epsilon_{x}$ into $v_{2}$. This is confirmed by studying the time evolution of the $v_{2}$, see Ref. [15] for details. 


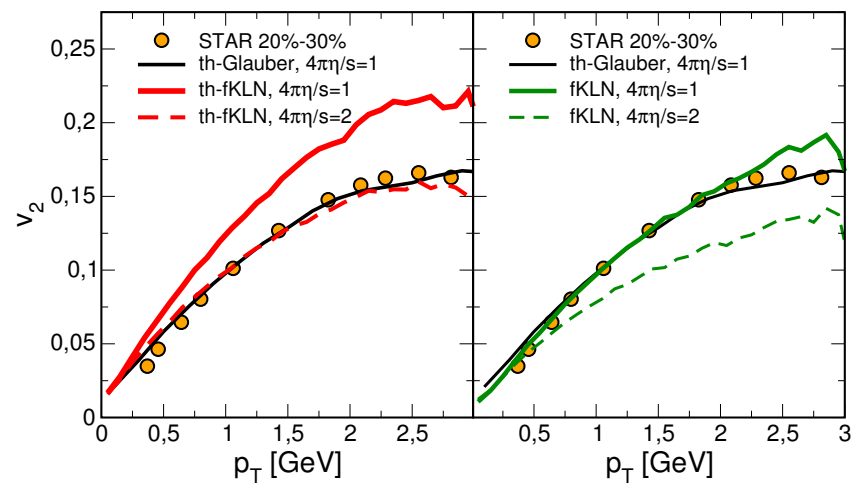

Figure 2. Elliptic flow $v_{2}\left(p_{T}\right)$ for the different initial conditions and $\eta / s$ as in the legend. All the calculations refer to Au-Au collisions at $\sqrt{s}=200 \mathrm{GeV}$, with an impact parameter $b=7.5 \mathrm{fm}$.

\section{Conclusions}

Our study based on kinetic theory shows that the elliptic flow in a system depends not only on the pressure gradients and the $\eta / s$ of the system, but also on the initial distribution in momentum space. A $p$-distribution with a saturation behavior generates smaller $v_{2}$ respect to the thermal one. In the specific case of the KLN matter studied here, the effect of the initial non-equilibrium distribution affects the estimate of $\eta / s$ of about a factor of two. The relevance of our results is further enhanced by the fact that Th-fKLN with $4 \pi \eta / s \sim 2$ would generate a low $v_{3}$ respect to the available data, which is the main conclusion of [14].

\section{References}

[1] B.V. Jacak, B. Muller, Science 337, 310 (2012)

[2] P. Romatschke, U. Romatschke, Phys.Rev.Lett. 99, 172301 (2007), 0706. 1522

[3] H. Song, U.W. Heinz, Phys.Rev. C78, 024902 (2008), 0805 . 1756

[4] L. Cifarelli, L. Csernai, H. Stocker, Europhys.News 43N2, 29 (2012)

[5] H. Song, S.A. Bass, U. Heinz, T. Hirano, C. Shen, Phys.Rev. C83, 054910 (2011), 1101.4638

[6] H. Niemi, G.S. Denicol, P. Huovinen, E. Molnar, D.H. Rischke, Phys.Rev.Lett. 106, 212302 (2011), 1101.2442

[7] G. Ferini, M. Colonna, M. Di Toro, V. Greco, Phys.Lett. B670, 325 (2009), 0805 . 4814

[8] Z. Xu, C. Greiner, H. Stocker, Phys.Rev.Lett. 101, 082302 (2008), 0711.0961

[9] E. Bratkovskaya, W. Cassing, V. Konchakovski, O. Linnyk, Nucl.Phys. A856, 162 (2011), 1101.5793

[10] S. Plumari, V. Greco, AIP Conf.Proc. 1422, 56 (2012), 1110.2383

[11] H.J. Drescher, Y. Nara, Phys.Rev. C75, 034905 (2007), nucl-th/0611017

[12] T. Hirano, Y. Nara, Phys.Rev. C79, 064904 (2009), 0904.4080

[13] S. Plumari, A. Puglisi, F. Scardina, V. Greco, Phys.Rev. C86, 054902 (2012), 1208.0481

[14] A. Adare et al. (PHENIX Collaboration), Phys.Rev.Lett. 107, 252301 (2011), 1105 . 3928

[15] M. Ruggieri, F. Scardina, S. Plumari, V. Greco (2013), 1303. 3178 